\title{
BMJ Open Developing a policy to reduce the salt content of food consumed outside the home in Malaysia: protocol of a qualitative study
}

\author{
Mhairi Karen Brown (D) , ${ }^{1}$ Suzana Shahar, ${ }^{2}$ Yee Xing You, ${ }^{2}$ Viola Michael, ${ }^{3}$ \\ Hazreen Abdul Majid, ${ }^{4}$ Zahara Abdul Manaf, ${ }^{2}$ Hasnah Haron, ${ }^{2}$ \\ Noor Shahida Sukiman, ${ }^{2}$ Yook Chin Chia, ${ }^{5,6}$ Feng J He, ${ }^{1}$ Graham A MacGregor ${ }^{1}$
}

To cite: Brown MK, Shahar S, You YX, et al. Developing a policy to reduce the salt content of food consumed outside the home in Malaysia: protocol of a qualitative study. BMJ Open 2021;11:e044628. doi:10.1136/ bmjopen-2020-044628

- Prepublication history and additional supplemental material for this paper is available online. To view these files, please visit the journal online (http://dx.doi org/10.1136/bmjopen-2020044628).

Received 08 September 2020 Accepted 15 June 2021
Check for updates

(C) Author(s) (or their employer(s)) 2021. Re-use permitted under CC BY-NC. No commercial re-use. See rights and permissions. Published by BMJ.

For numbered affiliations see end of article.

Correspondence to

Mhairi Karen Brown;

mhairi.brown@qmul.ac.uk

\section{ABSTRACT}

Introduction Current salt intake in Malaysia is high. The existing national salt reduction policy has faced slow progress and does not yet include measures to address the out of home sector. Dishes consumed in the out of home sector are a known leading contributor to daily salt intake. This study aims to develop a salt reduction strategy, tailored to the out of home sector in Malaysia.

Methods and analysis This study is a qualitative analysis of stakeholder views towards salt reduction. Participants will be recruited from five zones of Malaysia (Western, Northern, Eastern and Southern regions and East Malaysia), including policy-makers, non-governmental organisations, food industries, school canteen operators, street food vendors and consumers, to participate in focus group discussions or in-depth interviews. Interviews will be transcribed and analysed using thematic analysis. Barriers will be identified and used to develop a tailored salt reduction strategy.

Ethics and dissemination Ethical approval has been obtained from the Universiti Kebangsaan Malaysia Medical Research Ethics Committee (UKM PPI/1118/ JEP-2020-524), the Malaysian National Medical Research Ethics Committee (NMRR-20-1387-55481 (IIR)) and Queen Mary University of London Research Ethics Committee (QMERC2020/37) . Results will be presented orally and in report form and made available to the relevant ministries for example, Ministry of Health, Ministry of Education and Ministry of Trade to encourage adoption of strategy as policy. The findings of this study will be disseminated through conference presentations, peer-reviewed publications and webinars.

\section{INTRODUCTION}

Excess salt consumption is a leading risk factor for raised blood pressure, ${ }^{12}$ which in turn increases the risk of cardiovascular disease (CVD), the major cause of death and disability globally. ${ }^{3}$ Excess salt intake is also linked to stomach cancer, osteoporosis and kidney disease. ${ }^{4}$ The Global Burden of Disease Study estimates that suboptimal diets led to 8 million deaths in 2019 , with excess
Strengths and limitations of this study

- Our study is novel in that no country in Asia has yet developed an effective and comprehensive salt reduction strategy for food consumed outside the home, and our methodology will enable the collection of data that will enable the development of such a policy.

- Our recruitment strategy will ensure that we will gain views from a wide range of stakeholders across Malaysia including government, consumers and food vendors and manufacturers.

- The use of qualitative methodology with a wellestablished framework will allow us to address all concerns of stakeholders when developing the salt reduction strategy, ensuring support from all.

- The study will be conducted in Malaysia only, however, the findings could potentially be adapted by many other countries in the region to reduce salt in food consumed outside the home.

salt intake alone responsible for 2 million deaths. ${ }^{5}$

In 2013, World Health Organization (WHO) member states pledged to work towards a $30 \%$ relative reduction in population salt intake by $2025 .{ }^{6} \mathrm{WHO}$ has long highlighted salt reduction as a priority for population health, due to the impact and cost-effectiveness of the intervention, and recommends salt reduction as a 'best buy' intervention. ${ }^{7}$ The UK's salt reduction policy is often cited as a leading example, setting incrementally lower salt content targets for the food industry with independent monitoring, leading to a $15 \%$ reduction in population salt intake between 2003 and 2011 which was accompanied by a fall in average blood pressure and CVD mortality. ${ }^{8}$ Around 96 countries now have some form of a salt reduction policy in place, following the lead of the UK. ${ }^{9}$ However, global dietary salt intake 
remains much higher than the WHO recommended level of less than $5 \mathrm{~g}$ per day. ${ }^{10}$

In Malaysia, salt intake is also high $(7.9 \mathrm{~g} /$ day $)$ and hypertension is prevalent, particularly in adults from rural areas. ${ }^{11}{ }^{12}$ Indigenous ethnic groups are less likely to be aware of hypertension or to be treated, and those earning less than RM1000 have a forecasted higher level of 10-year CVD risk than those on higher incomes. ${ }^{1213}$ Therefore, salt reduction in Malaysia would benefit the entire community, particularly the more socially deprived. In 2015, the Malaysian Ministry of Health developed the Salt Reduction Strategy to Prevent and Control non-communicable diseases (NCD) for Malaysia (2015-2020), as part of their National Strategic Plan for NCDs (2016-2025), with the aim of achieving a $15 \%$ reduction in salt intake by $2020 .^{14}$ The salt reduction strategy includes measures to reformulate high salt products and increase awareness in the population of the impact salt has on health. However, an interim analysis found slow progress, and six recommendations were made to strengthen the strategy, which included a recommendation to address salt levels in the out-of-home sector, which is defined as anywhere food is consumed outside of the home, including but not limited to restaurants, street food vendors and hawkers, cafeterias, school canteens and hotels. ${ }^{15}$

Around three-quarters $(64.1 \%)$ of adults eat outside the home at least once a day in Malaysia. ${ }^{16} \mathrm{~A}$ baseline analysis found that out-of-home dishes are a leading contributor to daily salt intake, with dishes such as kolok mee, curry noodles and vegetables with soy/oyster sauce identified in the top five food sources. ${ }^{11}$ Foods sold in the out of home sector are often seasoned by consumers with a variety of sauces and condiments with high salt content but approximately half of these sauces and condiments do not have salt content printed on nutrition labels, making it difficult for consumers to evaluate how much salt they are adding to their food. ${ }^{17}{ }^{18}$ Instant noodles, a popular food choice among the younger population, have a mean salt content of $4.3 \pm 1.5 \mathrm{~g}$ per $100 \mathrm{~g}$ which is nearly four times higher than the salt content of food classified in Malaysia as a high salt content $(>1.2 \mathrm{~g}$ salt per $100 \mathrm{~g}) .{ }^{19}$ Evidence also shows that school canteen food and home cooked meals are a source of salt in the diets of children and adolescents. ${ }^{20}$ The main barriers for healthy eating at school identified by adolescents include the lack of healthy food options, the availability of unhealthy foods and issues around preferences and affordability. ${ }^{21}$ Furthermore, poor knowledge of the impact of salt on health and a lack of individual salt reduction practices contribute to high salt intake, even among healthcare staff in Malaysia. ${ }^{22}$

Food eaten outside the home is forming an increasing portion of daily diets in many countries, however, salt reduction strategies traditionally focus on processed packaged food, or awareness programmes, due to the availability of data on salt content printed on pack. ${ }^{1023}$ Data on the nutrition content of food served outside the home are lacking, particularly for street food vendors, and therefore, interviews will reveal potential barriers and solutions to addressing this.

The overall aim of this study is to develop a salt reduction strategy for the out of home sector in Malaysia. Given that Malaysia has a salt reduction strategy in place, as part of a wider strategy to reduce the burden of NCD in the country, it is likely that our strategy will be adopted by the Ministry of Health, the Ministry of Education and the Ministry of Trade and implemented as policy. ${ }^{11} 14$

Our objectives are:

1. Investigate the views of stakeholders across Malaysia towards salt reduction, to identify potential or perceived barriers.

2. Develop a strategy to reduce salt levels in out of home food, incorporating all themes from stakeholder discussions and present to government departments.

3. Conduct capacity building activities, including workshops, for chefs to increase awareness of salt reduction and methods.

\section{METHODS AND ANALYSIS}

\section{Overall study design}

A qualitative study will be conducted, using in-depth interviews and focus group discussions (FGD) to determine the views of stakeholders across Malaysia towards salt reduction, with an emphasis on the out of home sector. This design will enable participants to describe their views on potential perceived or actual barriers, allowing researchers to develop a tailored strategy and increasing likelihood of its adoption as policy by key decision-makers within the Ministry of Health, Ministry of Education and Ministry of Trade.

Qualitative research in this case is essential. While the methods of reducing salt in the population are well known, reducing salt in the out of home sector is a relatively new approach, without an existing successful model to replicate or adapt.

The study framework is illustrated in figure 1 and will use a social ecological model and the UK Medical Research Council framework, with adaptations from the Theoretical Domains Framework, creating a comprehensive framework that will capture a range of information required to develop a salt reduction strategy. ${ }^{24-26}$

\section{Study setting}

This study will be conducted across Malaysia, with participants recruited from five zones of the country to avoid a centralised and biased view. The target zones are:

- Western-Kuala Lumpur, Selangor, Perak.

- Southern-Johor, Melaka, Sembilan.

- Northern-Penang, Perlis, Kedah.

- Eastern-Pahang, Terengganu, Kelantan.

- East Malaysia-Sabah, Sarawak.

\section{Participants}

For this study, the sample was purposively selected and the sample size was determined based on the experience of similar studies, particularly a study in India. ${ }^{27}$ As this 


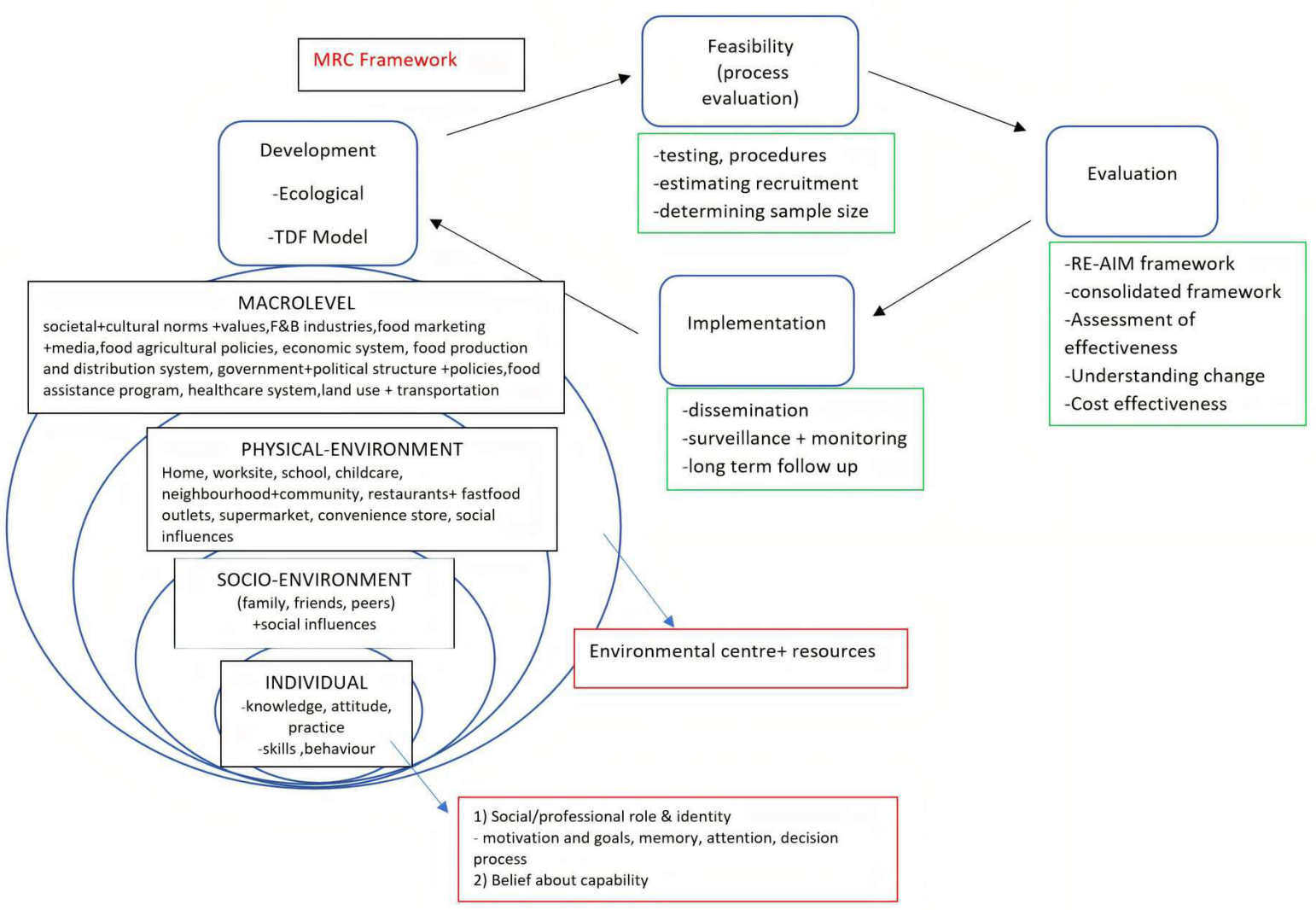

Figure 1 Study model developed by combining a social ecological model with adaptations from the UK Medical Research Council (MRC) framework and the Theoretical Domains Framework (TDF). RE-AIM, Reach, Effectiveness, Adoption, Implementation, Maintenance.

is a qualitative study, in-depth interviews and FGDs will continue until saturation of themes is achieved. ${ }^{28}$ We aim to recruit 285 participants. This is a large sample size for a qualitative study but the involvement of a wide range of stakeholders from several zones across Malaysia will ensure a strong and comprehensive strategy.

We will engage numerous stakeholders across Malaysia, including policy makers from the Ministry of Health, Ministry of Education and Ministry of Trade; nongovernmental organisations (NGOs); catering operators including the Indian Muslim Restaurants Association, school canteen operators and workplace cafeteria caterers; food industry representatives including soy sauce manufacturers and global food companies; street vendors and consumers (table 1 ).

\section{Recruitment}

Stakeholders will be recruited via email invitations, identified initially through existing contacts and networks and then moving to snowball recruitment.

Interested stakeholders will be screened for eligibility using inclusion and exclusion criteria and invited for an initial telephone meeting, where they will be given an overview of the study. Eligible participants will receive

Table 1 Study sampling frame

\begin{tabular}{llllllll}
\hline Group & Western & Southern & Northern & Eastern & East Malaysia & Sample size & Data collection method \\
\hline $\begin{array}{l}\text { Government, } \\
\text { policy and NGO }\end{array}$ & 10 & - & - & - & - & 10 & IDI \\
Caterers & 10 & 10 & 10 & 10 & 10 & 50 & IDI \\
Food industry & 5 & 5 & 5 & 5 & 5 & 25 & IDI \\
Street vendors & 5 & 5 & 5 & 5 & 5 & 25 & FGD \\
Consumers & 35 & 35 & 35 & 35 & 35 & 175 & FGD \\
\hline Total & 65 & 55 & 55 & 55 & 55 & 285 & \\
\hline
\end{tabular}

FGD, focus group discussion; IDI, in-depth interview; NGO, non-governmental organisation. 
Table 2 Inclusion and exclusion criteria

\begin{tabular}{|c|c|c|}
\hline Stakeholder group & Inclusion criteria & Exclusion criteria \\
\hline $\begin{array}{l}\text { Government, } \\
\text { policy and NGO } \\
\text { representatives }\end{array}$ & $\begin{array}{l}\text { Organisation with specific role in salt reduction of the population } \\
\text { Officer in charge with salt reduction/ food based programme/ policy } \\
\text { Officer working for the organisation for at least } 2-3 \text { years }\end{array}$ & \\
\hline Food industry & $\begin{array}{l}\text { Manufacturing foods identified as high salt sources } \\
\text { Officer in charge with industry's production planning/ policy } \\
\text { Officer working for the industry for at least } 2-3 \text { years } \\
\text { Include CEO/policy team plus nutrition teams to get a full view of the } \\
\text { support for salt reduction within the organisation, as well as input from } \\
\text { the key decision makers }\end{array}$ & \\
\hline Catering operators & $\begin{array}{l}\text { Indian Muslim Restaurants Association, Chef Association, franchised food } \\
\text { vendors, wedding caterers and/or school canteen operators } \\
\text { Operating food outlets at medium to large scale } \\
\text { Officer in charge with industry's production planning/ policy } \\
\text { Officer working for the industry for at least } 2-3 \text { years }\end{array}$ & \\
\hline
\end{tabular}

CEO, Chief Executive Officer; NGO, non-governmental organisation.

a written invitation to an FGD or in-depth interview as appropriate, with an information sheet and a consent form. Forms will be made available in Malay and English. Only those participants who have signed a consent form will be allowed to participate in interviews or FGDs.

The inclusion and exclusion criteria are as described in table 2.

For most stakeholder groups, we will aim to recruit participants who have been in post for 2-3 years to ensure they have the knowledge and experience to have an in-depth discussion or participate in an FGD. However, we will assess this on a case-by-case basis.

\section{Procedure}

Interview guides for the FGDs and in depth interviews have been developed (online supplemental appendix 1) and will be piloted with a minimum of five individuals who are not involved in the study but who belong to the target stakeholder groups. ${ }^{27}$ The pilot will take place in the Selangor (central) region where the majority of researchers involved are based. The interview guides will be refined following the pilot. All researchers involved in data collection will be given appropriate training before conducting FGDs and/or in-depth interviews.

Interviews will be conducted using the piloted interview guide, which consists of open-ended questions, allowing for a flexible approach that can be adapted depending on points raised and debate streams during the process. Interviews will be recorded using a digital voice recorder and researchers will also take notes during and after each interview or discussion to capture observations which may benefit the analysis.
Demographic and professional information will be collected. Interviews and discussions will take place during working hours in a comfortable and private room to help ensure open discussion. Interviews are expected to last 30-45 min, with FGDs lasting $60 \mathrm{~min}$.

Data collection has started, but due to COVID-19 restrictions a hybrid data collection has been adopted. The majority of the interviews with the Government departments and NGOs will be conducted online. Sessions with food industry representatives and consumers will be conducted face to face depending on approval by the local authorities.

\section{Data analysis}

Interviews and FGD will be transcribed verbatim and processed in QSR International NVivo V.11, a qualitative analysis software program. Inductive thematic analysis will be performed on transcribed interviews and discussions.

Using open coding, two researchers will code the transcripts and develop a coding framework which will be discussed and agreed by the research team. This will ensure consistent coding patterns. Themes and subthemes developed will be analysed critically and discussed among all researchers. A sample of the stakeholders involved in the interviews and discussions will be invited to read the findings and provide feedback to further refine the analysis.

A subsample of five transcribed interviews and/or discussions will be used for independent analysis by a researcher not involved in the interviews or coding to ensure the accuracy of developed themes and subthemes. We will randomly select 2-3 representatives from each stakeholder group for this purpose. 


\section{Salt reduction strategy development}

The themes and subthemes revealed through our study will be combined with existing knowledge of successful salt reduction interventions to develop a strategy to reduce salt levels in the out of home sector in Malaysia. All researchers will be involved in this process. Workshops will be organised to present various aspects of the strategy to relevant stakeholders to gain their feedback and strengthen the strategy.

In order to support implementation of the strategy as policy, researchers will also conduct capacity-building and knowledge-transfer activities, such as seminars, lecturers and practical workshops. The content of these activities will be designed to address the barriers raised during the study. Representatives from each of the stakeholder groups will be invited again to the capacity building activities.

\section{Project timelines}

Project timelines are illustrated in table 3 . The framework for the project was developed in March 2020, which informed development of the interview guides for FGDs and in-depth interviews. Ethics applications were submitted in June 2020, and participant recruitment began once ethical approval was obtained. It is anticipated that participant recruitment will begin from August 2020 to ending February 2021, with FGDs and in-depth interviews taking place from September 2020. With the current COVID-19 pandemic, interviews may be conducted virtually initially, with face-to-face interviews being conducted in 2021. Where interviews are conducted in person in 2020, national guidelines on social distancing and use of face masks will be adhered to.

Thematic analysis will take place in January 2021, with strategy development taking place from March 2021. Engagement with policy makers following strategy development is anticipated to begin in August 2021.

\section{Data management}

The study will be run in accordance with Good Clinical Practice. Data will be kept in accordance with the Data Protection Act, 1998. Physical materials such as consent forms and interview notes will be stored in locked cabinets at the research centre within UKM. Electronic data, including interview and discussion recordings and transcriptions will be stored in shared drives with a specific password access within UKM. Only appropriately trained research team members will have access to the data.

All participants will be assigned a coded number and pseudonym. No participant names or other identifying features will appear in any form of data reporting, with pseudonyms used to identify quotes or comments made by interviewees. The coding system will be stored digitally and manually at UKM and will only be able to be accessed by a member of the research team. Data will be held securely for 10 years, according to Universiti Kebangsaan Malaysia Medical Research Ethics Committee policy.
All participant-identifiable data linked to the participant via their unique ID will be kept separately from anonymised research data in a password-protected file. Electronic data will only be stored with participant ID in a password-protected file on secure UKM servers.

\section{Patient and public involvement}

While developing the funding application for this project, we approached the Ministry of Health Malaysia to present our plans and gain their feedback to strengthen our application. Their experience of overseeing salt reduction in Malaysia was beneficial to help us define the food industry representatives and other stakeholders crucial to involve in this project. We also gained their approval to be involved in interviews and dissemination of the results, as they will facilitate dissemination to other Government departments and their network of food industry representatives.

\section{Ethics and dissemination}

Ethical approval has been obtained from the Universiti Kebangsaan Malaysia Medical Research Ethics Committee (reference UKM PPI/111/8/JEP-2020-524), the Malaysian National Medical Research Ethics Committee (reference NMRR-20-1387-55481 (IIR)) and Queen Mary University of London Research Ethics Committee (reference QMERC2020/37). There are no substantive ethical issues associated with the conduct of our research project.

Written informed consent will be obtained from all participants. Every participant has the right to withdraw from the study at any time and participation is solely on a voluntary basis.

Pseudonyms will be allocated in all interviews and transcriptions of data will be anonymised, to ensure confidentiality. Participants' identifiable information will only be used for the purposes of arranging interviews and obtaining signed consent. Demographic data will be aggregated among participants and compiled in tables. Records will be stored securely on a password protected computer and paper copies of the consent form will be stored separately in a locked cabinet, only accessible by the researchers.

Results will be presented orally and in report form and made available to Ministry of Health, Ministry of Education and Ministry of Trade staff to encourage adoption of strategy as policy.

The findings of this study will be disseminated through conference presentations and peer-reviewed publications, as well as via webinars, newsletters and press releases through the World Action on Salt, Sugar and Health (WASSH) network of more than 600 international experts. $^{29}$

Findings from this study will contribute to the wider salt reduction strategy in Malaysia, and will inform global salt reduction efforts. It is anticipated that our findings will also inform and improve the School Health Programme. We also anticipate that as a result of our study, the Ministry of Trade will support those manufacturers producing 


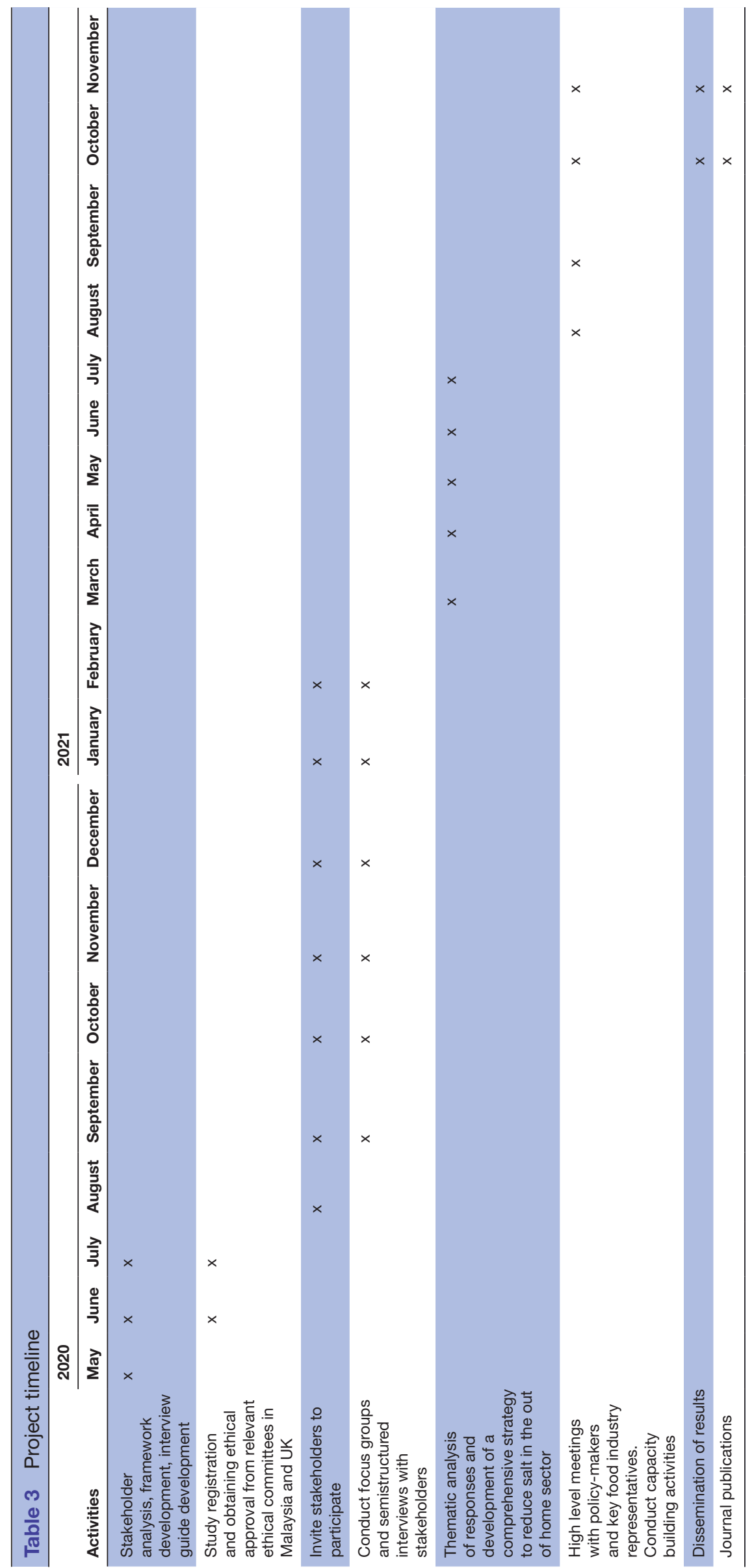

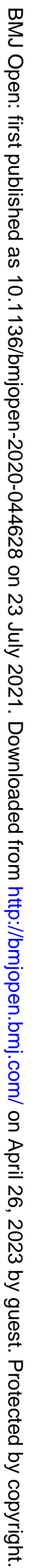


more healthy product portfolios. In addition, as school children buy food products from school canteen operators, our findings will benefit and strengthen healthy eating and salt reduction work by both the Ministry of Education and the Ministry of Health. Strengthening collaborative work is essential to ensure the food environment is healthy for children.

\section{COVID-19}

Malaysia has experienced several waves of the COVID-19 pandemic and restrictions are currently in place to help prevent further infections. A Movement Control Order (MCO) is applied as necessary to the whole country or to specific states, depending the number of active COVID-19 cases.

The MCO has impacted our project, specifically interstate or interdistrict travelling and in-person group meetings. Data collection and meetings involving policymakers, NGOs and researchers could be conducted effectively through online, virtual interviews, but interviews and FGD with food industry representatives and street food vendors would be best conducted in person and accompanied by a visit to the respective factories that manufacture products sold by companies and used by street food vendors. Project timelines will be monitored and adapted to ensure that in-person interviews and FGDs can be conducted later in the year when the MCO is likely to be lifted.

\section{Author affiliations \\ ${ }^{1}$ Wolfson Institute of Preventive Medicine, Barts and The London School of Medicine and Dentistry, Queen Mary University of London, London, UK \\ ${ }^{2}$ Faculty of Health Sciences, Universiti Kebangsaan Malaysia, Kuala Lumpur, Malaysia \\ ${ }^{3}$ Disease Control Division, Ministry of Health, Putrajaya, Malaysia ${ }^{4}$ Centre for Population Health (CePH) and Department of Social and Preventive Medicine, Faculty of Medicine, University Malaya, Kuala Lumpur, Malaysia ${ }^{5}$ Department of Medical Science, Sunway University, Bandar Sunway, Malaysia ${ }^{6}$ Department of Primary Care Medicine, University of Malaya, Kuala Lumpur, Malaysia}

Contributors FJH, SS, HAM, MKB and GAM conceived the project and designed the study. MKB wrote the first draft of the manuscript. SS, YXY, VM, HAM, ZAM, NSS, YCC and HH designed the questionnaires. All authors contributed to the refinement of the study protocol and approved the final manuscript.

Funding This work is supported as part of the Newton Fund Impact Scheme by the Medical Research Council on behalf of UK Research and Innovation in the UK and by the Malaysia Industry-Government Group for High Technology (MiGHT) (grant number: MR/V005847/1). The views expressed are those of the authors and not necessarily those of the MRC or MiGHT.

Competing interests None declared.

Patient consent for publication Not required.

Provenance and peer review Not commissioned; externally peer reviewed.

Supplemental material This content has been supplied by the author(s). It has not been vetted by BMJ Publishing Group Limited (BMJ) and may not have been peer-reviewed. Any opinions or recommendations discussed are solely those of the author(s) and are not endorsed by BMJ. BMJ disclaims all liability and responsibility arising from any reliance placed on the content. Where the content includes any translated material, BMJ does not warrant the accuracy and reliability of the translations (including but not limited to local regulations, clinical guidelines, terminology, drug names and drug dosages), and is not responsible for any error and/or omissions arising from translation and adaptation or otherwise.
Open access This is an open access article distributed in accordance with the Creative Commons Attribution Non Commercial (CC BY-NC 4.0) license, which permits others to distribute, remix, adapt, build upon this work non-commercially, and license their derivative works on different terms, provided the original work is properly cited, appropriate credit is given, any changes made indicated, and the use is non-commercial. See: http://creativecommons.org/licenses/by-nc/4.0/.

ORCID iD

Mhairi Karen Brown http://orcid.org/0000-0001-6991-700X

\section{REFERENCES}

1 He FJ, Li J, Macgregor GA. Effect of longer term modest salt reduction on blood pressure: cochrane systematic review and metaanalysis of randomised trials. BMJ 2013;346:f1325.

2 Huang L, Trieu K, Yoshimura S, et al. Effect of dose and duration of reduction in dietary sodium on blood pressure levels: systematic review and meta-analysis of randomised trials. BMJ 2020;368:m315.

3 World Health Organization. Cardiovascular diseases (CVD), 2017.

$4 \mathrm{He}$ FJ, Tan M, Ma Y, et al. Salt reduction to prevent hypertension and cardiovascular disease: JACC state-of-the-art review. J Am Coll Cardiol 2020;75:632-47.

5 GBD 2019 Risk Factors Collaborators. Global burden of 87 risk factors in 204 countries and territories, 1990-2019: a systematic analysis for the global burden of disease study 2019. Lancet 2020;396:1223-49.

6 World Health Organization. Global action plan for the prevention and control of noncommunicable diseases 2013-2020. Geneva, 2013.

7 World Health Organization. Best buys' and other recommended interventions for the prevention and control of noncommunicable diseases, updated (2017) appendix III of the global action plan for the prevention and control of non-communicable diseases 2013-2020. Geneva, Switzerland, 2017.

8 He FJ, Pombo-Rodrigues S, Macgregor GA. Salt reduction in England from 2003 to 2011: its relationship to blood pressure, stroke and ischaemic heart disease mortality. BMJ Open 2014;4:e004549.

9 Santos JA, Tekle D, Rosewarne E, et al. A systematic review of salt reduction initiatives around the world: a midterm evaluation of progress towards the 2025 global non-communicable diseases salt reduction target. Adv Nutr 2021. doi:10.1093/advances/nmab008. [Epub ahead of print: 07 Mar 2021].

10 Trieu K, Neal B, Hawkes C, et al. Salt Reduction Initiatives around the World - A Systematic Review of Progress towards the Global Target. PLoS One 2015;10:e0130247.

11 Institute for Public Health (IPH). Population-Based salt intake survey to support the National salt reduction programme for Malaysia (Malaysian community salt survey - MyCoSS).

12 Abdul-Razak S, Daher AM, Ramli AS, et al. Prevalence, awareness, treatment, control and socio demographic determinants of hypertension in Malaysian adults. BMC Public Health 2016;16:351.

13 Su TT, Amiri M, Mohd Hairi F, et al. Prediction of cardiovascular disease risk among low-income urban dwellers in metropolitan Kuala Lumpur, Malaysia. Biomed Res Int 2015;2015:516984.

14 Ministry of Health Malaysia. National strategic plan for noncommunicable disease (NSPNCD) 2016-2025. Putrajaya, Malaysia, 2016.

15 Ministry of Health Malaysia. Reducing salt intake in Malaysia: an interim evaluation of what works. Putrajaya, Malaysia, 2019.

16 Poulain J-P, Laporte $C$, Tibère L, et al. Malaysian food barometer (MFB): a study of the impact of compressed modernisation on food habits. Malays J Nutr 2020;26:001-17.

17 Shahar S, You YX, Zainuddin NS, et al. Sodium content in sauces-a major contributor of sodium intake in Malaysia: a cross-sectional survey. BMJ Open 2019;9:e025068

18 Haron $\mathrm{H}$, Hiew I, Shahar S, et al. A survey on salt content labeling of the processed food available in Malaysia. Int J Environ Res Public Health 2020;17:2469.

19 Tan $\mathrm{CH}$, Chow ZY, Ching SM, et al. Salt content of instant noodles in Malaysia: a cross-sectional study. BMJ Open 2019;9:e024702.

20 Abdul Majid H, Ramli L, Ying SP, et al. Dietary intake among adolescents in a middle-income country: an outcome from the Malaysian health and adolescents longitudinal research team study (the MyHeARTs study). PLoS One 2016;11:e0155447.

21 Mohammadi S, Su TT, Papadaki A, et al. Perceptions of eating practices and physical activity among Malaysian adolescents in secondary schools: a qualitative study with multi-stakeholders. Public Health Nutr 2021;24:2273-85.

22 Mahat DIZ, Tamil AM, Mahmood MI, et al. The association of knowledge, attitude and practice with 24 hours urinary sodium 
excretion among Malay healthcare staff in Malaysia. International Journal of Public Health Research 2017;7:860-70.

23 World Bank Blogs. World bank blogs, 2015. Available: https://blogs. worldbank.org/developmenttalk/more-people-developing-world-areeating-out-measuring-well-could-change-our-understanding-poverty

24 Medical Research Council. Developing and evaluating complex interventions.

25 Phillips CJ, Marshall AP, Chaves NJ, et al. Experiences of using the theoretical domains framework across diverse clinical environments: a qualitative study. $J$ Multidiscip Healthc 2015;8:139-46.
26 Story M, Kaphingst KM, Robinson-O'Brien R, et al. Creating healthy food and eating environments: policy and environmental approaches. Annu Rev Public Health 2008;29:253-72.

27 Gupta P, Mohan S, Johnson C, et al. Stakeholders' perceptions regarding a salt reduction strategy for India: findings from qualitative research. PLoS One 2018;13:e0201707.

28 Saunders B, Sim J, Kingstone T, et al. Saturation in qualitative research: exploring its conceptualization and operationalization. Qual Quant 2018;52:1893-907.

29 World Action on Salt Sugar and Health. WASSH network. Available: http://www.worldactiononsalt.com/about/history/\#d.en.484285 